\title{
ESTADO NUTRICIONAL, FERRO, COBRE E ZINCO EM ESCOLARES DE FAVELAS DA CIDADE DE SÃO PAULO
}

\author{
Elisabete B. Santos, Olga M. S. Amancio*, Carlos A.G. Oliva \\ Trabalho realizado no Departamento de Pediatria da Universidade Federal de São Paulo
}

\section{*Correspondência}

Rua Botucatu, 703

Cep: 04023-062

São Paulo/ SP

omsamancio.dped@epm.br

\begin{abstract}
RESUMO
Oвjetrvo. Avaliar a antropometria, a composição corporal e o estado nutricional em ferro, cobre e zinco segundo o gênero, de crianças e adolescentes institucionalizados, moradores de duas favelas da cidade de São Paulo.

Métodos. Estudo transversal utilizando medidas de peso, estatura, circunferência braquila, dobras cutâneas, bioimpedância elétrica, os escores Z da relação estatura para idade, índice de massa corporal, área do braço, área muscular do braço e área de gordura do braço. Os percentuais de gordura corporal e massa magra foram analisados segundo fórmulas de Siri e Slaughter. Foram determinados hemoglobina, hematócrito, ferro, ferritina, cobre e zinco séricos.

REsultados. Foram maiores o peso corporal, circunferência do braço, dobras cutâneas do tríceps e subescapular, resistência elétrica, escores $Z$ da área do braço, área muscular do braço e percentual de gordura corporal no sexo feminino em relação ao masculino. Baixa estatura foi encontrada em $8 \%$ das meninas e 5,6\% dos meninos, sem diferença quanto ao gênero. Houve menor prevalência de desnutrição ( $2 \%$ das meninas e $5,6 \%$ nos meninos), do que de sobrepeso e obesidade ( $30 \%$ e $11,2 \%$, respectivamente). Observou-se anemia em $24,4 \%$ e ferropenia em 10,5\% dos escolares com ou sem anemia. Apresentaram valores abaixo do limite inferior do padrão de referência para cobre e zinco séricos, respectivamente três e sete indivíduos.
\end{abstract}

Conclusão. Na população estudada, de baixo nível econômico e institucionalizada, ocorre o processo de transição nutricional e alta prevalência de anemia que não resulta da interação ferro, cobre e zinco.

Unitermos: Estado nutricional. Composição corporal. Ferro. Cobre. Zinco.

\section{INTRODUÇÃO}

Segundo dados da Organização Mundial de Saúde (OMS), a prevalência de comprometimento grave do crescimento em países em desenvolvimento tem diminuído progressivamente de $47 \%$ em 1980 para 33\% em 2000. Cerca de um terço de todas as crianças com idade inferior a 5 anos apresentam comprometimento grave do crescimento, sendo que $70 \%$ vivem na Ásia, 26\% na África e 4\% na América Latina e Caribe!.

O "stunting" nutricional na infância, um indicador de desnutrição crônica, tem sido sugerido como um fator que contribui para altas taxas de obesidade em adolescentes e adultos de países em desenvolvimento ${ }^{2}$.

A comparação de estudos realizados nos períodos de 1974/ 75 e 1995/96 sobre a prevalência de desnutrição e obesidade em crianças e adolescentes, mostra que em nosso país a desnutrição diminuiu de $14,8 \%$ para $8,6 \%$, sendo que a obesidade aumentou de $4,1 \%$ para $13,9 \%^{3}$.

Assim, a progressão da transição nutricional caracterizada por redução na prevalência dos déficits nutricionais e a ocorrência mais expressiva de sobrepeso e obesidade têm sido observadas em sociedades em desenvolvimento que experimentam rápidas e intensas transformações de crescimento econômico e de estrutura demográfica ${ }^{4}$.
O uso de índices antropométricos tem sido uma estratégia válida para gerar indicadores sensíveis do estado nutricional, tendo como objetivo determinar a massa corporal, expressa pelo peso; as dimensões lineares, especialmente a estatura; e a composição corporal (a gordura subcutânea e a massa muscular). Seus resultados podem refletir, também, a condição de vida dos grupos populacionais estudados 5 .

Além do déficit pôndero-estatural e da vulnerabilidade às doenças infecto-parasitárias, a desnutrição, quer por insuficiência quer por excesso, se correlaciona com outras carências nutricionais como de micronutrientes.

A anemia por deficiência de ferro é atualmente o problema nutricional mais prevalente, afetando cerca de 1,3 bilhão de pessoas, com aumento da sua prevalência e morbidade em crianças e adolescentes do sexo feminino ${ }^{6}$. Diferencia-se de outras condições carenciais na medida em que sua ocorrência não se limita apenas às populações de baixa renda, desnutridos ou indivíduos submetidos a condições especiais de risco, mas acomete também nos grupos mais favorecidos e de condição global adequada?

Outras deficiências podem ocorrer em função da maior necessidade para o crescimento, como a de cobre e de zinco que fazem parte da estrutura das metaloenzimas e agem como co-fatores enzimáticos, estando, pois, envolvidas em diversas funções me- 
tabólicas, inclusive as inerentes ao crescimento linear e a composição corporal .

Considerando o exposto, objetivou-se investigar a antropometria, a composição corporal e o estado nutricional em ferro, cobre e zinco, segundo o gênero, de crianças e adolescentes institucionalizados, moradores de duas favelas da cidade de São Paulo.

\section{Métodos}

Trata-se de estudo transversal, com 86/140 escolares de 7 a 15 anos, moradores de duas favelas, Rocinha e Beira Rio, da zona sul da cidade de São Paulo, que freqüentavam o Centro de Juventude da Associação dos Cavaleiros da Soberana Ordem Militar de Malta de São Paulo e Brasil/Meridional, entidade internacional não-governamental. Este trabalho foi aprovado pelo Comitê de Ética em Pesquisa da Universidade Federal de São Paulo, tendo sido necessário o consentimento prévio por escrito dos pais ou responsáveis.

\section{Critérios de inclusão}

Ausência de hospitalização nos últimos 30 dias; ausência de doenças crônicas conhecidas; sem uso de suplementação de ferro, que interferiria nos resultados bioquímicos?

Os indivíduos estudados foram divididos em dois grupos, segundo o gênero.

\section{Avaliação do nível econômico}

Foi determinado pela renda mensal em salários mínimos per capita $^{10}$

\section{Avaliação antropométrica}

Peso e estatura: a tomada de dados foi realizada segundo técnicas padronizadas". Para o cálculo do escore $Z$ da relação estatura para a idade (E/l), foi utilizado o programa Epi Info' ${ }^{2}$.

Índice de Massa Corporal (IMC): de acordo com os valores de IMC obtidos, os indivíduos foram classificados em eutróficos, desnutridos, sobrepeso e obesos ${ }^{13}$. Calculou-se o escore Z do Índice de Massa Corporal.

Circunferência braquial: esta medida foi realizada de acordo com a técnica de Frisancho ${ }^{14}$.

Dobras cutâneas: para mensuração das dobras cutâneas foi utilizado Lange Skinfold Caliper. Foram feitas três medidas consecutivas, calculando-se a média entre elas. Foram avaliadas as dobras cutâneas do tríceps, bíceps, supra-ilíaca e subescapular.

A medida da circunferência braquial foi convertida em mm, para o cálculo da área do braço (AB), área muscular do braço (AMB) e área de gordura do braço (AGB) segundo Frisancho'14. Foram calculados os escores $Z$ de cada variável utilizando valores de referência de Frisancho ${ }^{15}$.

Bioimpedância elétrica: a bioimpedância elétrica foi realizada por meio do aparelho RJL System, modelo I0I, com o indivíduo em jejum por pelo menos duas horas. Os eletrodos foram colocados de acordo com o preconizado ${ }^{16}$. Foram realizadas três medidas consecutivas, adotando-se a média entre elas.

\section{Análise da composição corporal}

Para a análise da composição corporal foram calculadas a massa gorda e a massa magra em quilogramas $(\mathrm{kg})$ e em percentual do peso corporal (\%), segundo metodologias de Siri $^{17}$ e Slaughter et al. ${ }^{18}$. A densidade corporal foi estimada por meio das fórmulas propostas por Brook ${ }^{19}$. Os percentuais de massa gorda e magra foram comparados com o padrão de referência de Fomon et al. ${ }^{20}$ em crianças até 10 anos de idade e de Haschke ${ }^{21}$ em crianças maiores de 10 anos.

\section{Avaliação bioquímica}

Para as determinações bioquímicas foram coletados $8 \mathrm{~mL}$ de sangue por meio de seringas e agulhas descartáveis, estando os participantes em jejum de pelo menos três horas.

Hemoglobina e hematócrito: as dosagens de hemoglobina e hematócrito foram realizadas em aparelho automatizado Cell-Dyn 3800. Considerou-se anemia os níveis de hemoglobina $<11,5 \mathrm{~g} /$ $\mathrm{dL}$ (crianças entre $5 \mathrm{e} \mid 1$ anos); < $12 \mathrm{~g} / \mathrm{dL}$ (entre 12 e 14 anos, e meninas acima de 15 anos) $)^{22}$.

Ferro e ferritina séricos: o ferro sérico foi determinado utilizando o kit Ironâ da Bayer. A ferritina foi determinada pelo kit Immulite - Ferritinâ da Diagnostic Products Corporation.

Apesar de não existir completa uniformidade na literatura, considerou-se indicativos de deficiência de ferro: ferro sérico < $50 \mathrm{mg} / \mathrm{dL}$; ferritina sérica $<12 \mathrm{ng} / \mathrm{mL}^{23}$.

Cobre e zinco séricos: as amostras de soro foram diluídas em água Milli-Q, na proporção de 1:10 e as concentrações de cobre e zinco foram determinadas por espectrofotometria de absorção atômica ("Perkin Elmer" modelo 5.100) nas condições especificadas pelo fabricante. Os resultados foram expressos como $\mathrm{mg} \mathrm{Cu}$ e $\mathrm{Zn} / \mathrm{dL}$. Adotou-se valores de referência para o sexo masculino de 70 a $140 \mathrm{mg} \mathrm{Cu} / \mathrm{dL}$ e de 65 a $120 \mathrm{mg} \mathrm{Zn} / \mathrm{dl}$, e para o sexo feminino de 80 a $155 \mathrm{mg}$ Cu/dL e de 60 a $120 \mathrm{mg} Z \mathrm{Zn} / \mathrm{dL}^{24}$.

\section{Análise estatística}

Teste $t$ de Student e teste de Mann-Whitney para comparação entre dois grupos, de variáveis quantitativas de distribuição paramétrica e não-paramétrica, respectivamente.

Teste de Qui-quadrado ou teste exato de Fisher, para comparação das freqüências de variáveis qualitativas. Adotou-se a $<0,05$.

\section{Resultados}

O número mediano de pessoas por família foi cinco e a renda per capita foi inferior a 0,5 salário mínimo em 79\% dos indivíduos, sendo que apenas um apresentou renda per capita entre I,5 e 2,5 salários mínimos (dados não mostrados).

Embora não houvesse diferença significante entre os gêneros quanto à média da estatura $(p=0,12)$ (dado não mostrado) e quanto à média do escore $Z E / I(p=0,27)$ (Tabela I), nos dois 


\begin{tabular}{lcccc}
\hline \multicolumn{4}{c}{ Tabela I-Valores médiose medianos das variáveis nutricionais do grupo } \\
estudado, segundo ogênero
\end{tabular}

\footnotetext{
${ }^{a}$ media; ${ }^{b}$ mediana

( ) variação dos valores encontrados

I nivel descritivo do teste $t$ de Student

2 nível descritivo do teste de Mann-Whitney

3 nível descritivo do teste do Qui-quadrado
}

gêneros a distribuição dos valores de estatura ocorreu abaixo do percentil 50 do padrão de referência, verificando-se baixa estatura em 4/50 (8,0\%) das meninas e 2/36 (5,6\%) dos meninos (teste do Qui-quadrado, $p=0,50$ ).

Foram significantemente maiores no sexo feminino as médias de peso corporal, da circunferência do braço, das dobras cutâneas do tríceps e subescapular e da resistência elétrica, respectivamente $p=0,0$ l (dados não mostrados). Em relação ao peso corporal, no sexo feminino os valores estavam distribuídos acima do percentil 50 do padrão de referência, enquanto no masculino essa distribuição ocorreu abaixo desse percentil.

O sexo feminino apresentou valores maiores de escores $Z$ do IMC, $A B$ e AMB, assim como a freqüência de sobrepeso e obesidade (Tabela I). Quanto ao percentual de gordura corporal, o sexo feminino apresentou percentuais maiores do que o sexo masculino tanto pelo método de Siri $(p=0,0 \mathrm{I})$ como pelo de Slaughter $(p<0.0 \mathrm{I})$ (dados não mostrados).

A prevalência de anemia, em ambos os gêneros, foi de 24,4\% (2I/86) e os níveis séricos de ferro e ferritina não apresentaram diferença segundo o gênero (Tabela 2).

No sexo feminino, 24 (48\%) apresentaram níveis de cobre sérico acima do limite superior do padrão de referência e 2 (4\%) abaixo do limite inferior; enquanto que no sexo masculino esses números foram $12(33,4 \%)$ e I (2,8\%), respectivamente. A mediana do gênero feminino foi maior que a do masculino $(p=0,04)$ (Tabela 2).

Quanto aos valores de zinco sérico (Tabela 2) observou-se médias de acordo com o padrão de referência nos dois gêneros e sem diferença significante entre eles $(p=0,54)$, porém seis indivíduos, um do gênero feminino, apresentaram valores abaixo do limite inferior do padrão de referência.

\begin{tabular}{lccc}
\hline \multicolumn{4}{c}{ Tabela 2-Valores hematológicos e séricos do grupo estudado, } \\
segundo o gênero
\end{tabular}

(I) t de Student

(2) Mann-Whitney

(3) Qui-quadrado

( ) variação dos valores encontrados

[ ] valores de referência (Gibson, 1990)

Tabela 3-Valores médios de ferro sérico (mg/dL) no grupo estudado, segundo a presença ou não de anemia

\begin{tabular}{|c|c|c|}
\hline & Ferro sérico $(\mu \mathrm{g} / \mathrm{dL})$ & $\mathrm{Fe}<50(\mu \mathrm{g} / \mathrm{dL})$ \\
\hline Anemia & $\mathrm{x} \pm \mathrm{dp}$ & $n(\%)$ \\
\hline Não & $86,4 \pm 28,1$ & $4(6,2)$ \\
\hline Sim & $72,0 \pm 28,0$ & $5(23,8)$ \\
\hline Valor de $p$ & $0,04^{(I)}$ & $0,03^{(2)}$ \\
\hline
\end{tabular}

(2) teste exato de Fisher

As crianças e adolescentes com anemia tiveram níveis séricos de ferro menores $(p=0,04)$ e maior incidência de ferropenia $(p=$ 0,03 ) do que aqueles sem anemia (Tabela 3).

Não houve diferença significante entre as médias de cobre $(p=0,22)$ e de zinco $(p=0,08)$ séricos segundo a presença de anemia (dados não mostrados).

\section{Discussão}

Os resultados da renda per capita estão de acordo com o Instituto Brasileiro de Geografia e Estatística (IBGE) ${ }^{25}$, que relata apenas 2,4\% da população com renda até 0,5 salário mínimo no estado de São Paulo.

A incidência de baixa estatura verificada em ambos os gêneros, provavelmente se deve à desnutrição pregressa nos primeiros anos de vida. A literatura registra em crianças e adolescentes dos 
sexos feminino e masculino e de baixa renda, estatura menor em relação aos de maior renda ${ }^{26}$.

Verificou-se baixa prevalência de desnutrição, 2\% nas meninas e 5,6\% nos meninos, sendo que as meninas apresentaram maior freqüência de sobrepeso e obesidade (30\%) do que os meninos $(\mathrm{I}, 2 \%)$. Esse resultado pode estar relacionado com o processo de transição nutricional que vem ocorrendo em grupos de baixo nível econômico de forma semelhante aos de maior renda. Vários estudos $^{27-28}$ relatam a transição nutricional em crianças e adolescentes, inclusive com significância no gênero feminino em relação ao masculino, confirmando os resultados obtidos neste trabalho.

Quanto à composição corporal, o sexo feminino apresentou percentual de gordura corporal maior que o masculino, bem como a circunferência do braço, dobras cutâneas triciptal e subescapular, resistência elétrica, área do braço e área muscular do braço. Alguns autores relatam maior percentual de gordura corporal2 ${ }^{29}$, dobras cutâneas e circunferência do braço em menina ${ }^{30}$. Há estudos indicando que atualmente crianças e adolescentes apresentam maior quantidade de gordura corporal que as de gerações passa$\mathrm{das}^{31-32}$. Nesse sentido, a implicação maior é que, seguindo esta tendência, pode-se identificar mudanças no curso de vida destes indivíduos, com concomitante aumento na prevalência de doenças crônicas não transmissíveis e conseqüentemente, aumento na carga para os serviços de saúde.

Para se compreender os principais determinantes de sobrepeso e da obesidade na infância e adolescência, a literatura tem discutido amplamente as práticas alimentares inadequadas, o tempo de inatividade, sobrepeso e obesidade dos pais, peso ao nascer e também as condições socioeconômicas das famílias ${ }^{33}$. A obesidade tem-se associado a fatores ambientais e comportamentais, que interagem de forma complexa. Sobrepeso e obesidade têm se relacionado de forma inconsistente com padrões socioeconômicos, etnia e gênero, não havendo um padrão definido de associação que seja generalizáve ${ }^{34}$.

Em indivíduos desnutridos e com excesso de peso ocorrem deficiências de micronutrientes, sendo que a mais prevalente é a anemia por deficiência de ferro. Monteiro et al. ${ }^{35}$, estimando a prevalência da anemia na infância na cidade de São Paulo, constatam que no espaço de dez anos houve redução significativa na concentração média de hemoglobina com aumento na prevalência de anemia de $35,6 \%$ para $46,9 \%$, atingindo meninas e meninos principalmente dos grupos de menor renda. Segundo a OMS22 a prevalência de anemia em crianças e adolescentes de países em desenvolvimento está em torno de $48,1 \%$ e de $5,9 \%$ em países desenvolvidos.

Apesar do grupo estudado freqüentar uma instituição social, a prevalência de anemia em ambos os gêneros $(24,4 \%)$ e de ferropenia nos indivíduos com anemia $(23,8 \%)$ pode estar relacionada com ingestão insuficiente de ferro e o fato de morarem em favelas poder desencadear a ocorrência de parasitismo. Embora alta, a prevalência de anemia nos indivíduos estudados mostrou-se menor do que a observada anteriormente, $53,5 \% \%^{36}$. O fato de não ter sido observada diferença nos valores de $\mathrm{Hb}$ entre os gêneros confirma relato de Norton et al. ${ }^{37}$ para a mesma faixa etária.
Os valores de ferro sérico sem diferença entre os gêneros confirmam resultados de Alárcon et al. ${ }^{38}$. Neste parâmetro a variação foi de 24,0 a $177,0 \mathrm{mg} / \mathrm{dL}$, sendo que $10,5 \%$ apresentaram ferropenia. Diferentemente dos resultados obtidos neste trabalho, Nead et al. ${ }^{39}$ não verificam diferença na prevalência de deficiência de ferro nas crianças e adolescentes com ou sem anemia.

Em relação ao cobre sérico, Alárcon et al. ${ }^{38}$ também relatam maiores concentrações séricas de cobre no sexo feminino do que no masculino. Há relatos de aumento dos níveis séricos de cobre em meninas com idades entre 12 e 19 anos ou 15 e 18 anos $^{40-41}$ quando comparado com os meninos. No presente estudo verificou-se baixa prevalência de deficiência de cobre (3,48\%). Este baixo percentual pode ser explicado, por se tratarem de crianças e adolescentes institucionalizados, pressupondo-se que a alimentação fornecida pela instituição social consiga fornecer as quantidades necessárias diárias deste mineral. Estévez et al. ${ }^{40}$ descrevem prevalência de deficiência de cobre em $7 \%$ de indivíduos não institucionalizados.

A concentração de zinco sérico nas meninas e meninos na infância é baixa, alcançando um pico na adolescência e declinando na idade adulta. Laitinen et al. ${ }^{41}$ verificam que os níveis séricos de zinco não diferem aos 3, 6, 9 e 12 anos, sendo que os meninos apresentam nas idades de 15 e 18 anos valores maiores em relação ao das meninas. Porém, outros estudos avaliando a concentração de zinco sérico em crianças e adolescentes não observam diferença quanto ao gênero ${ }^{38,40}$ confirmando o resultado do presente estudo.

A prevalência de deficiência de zinco no presente estudo foi baixa (6,9\%) quando comparada aos estudos de Estévez et al..$^{40}$ que relatam deficiência em 19,6\% da população, enquanto Favaro, $V_{\text {annucchi }}{ }^{22}$ descrevem em 14\% nas meninas e 11,7\% nos meninos. A população estudada por esses autores corresponde também a indivíduos de baixa renda, porém não institucionalizados, o que pode estar relacionado com os altos índices observados por esses autores.

Entre os indivíduos com ou sem anemia, não houve diferença para o cobre e zinco séricos. Esse resultado mostra que não houve interação entre zinco, cobre e ferro, situação na qual teria ocorrido anemia por alta ingestão de zinco, ou seja, alta ingestão de zinco dietético levando à deficiência de cobre por competição pelos sítios de ligação da metalotioneína nas células mucosas intestinais ${ }^{43}$, que por sua vez restringe a utilização de ferro. A ceruloplasmina, proteína cobre dependente, é essencial para a mobilização de ferro a partir dos depósitos, antes de sua incorporação na hemoglobina ${ }^{44}$. $\mathrm{Na}$ deficiência de cobre, a ceruloplasmina tem sua atividade significantemente diminuída, levando à anemia, a qual é responsiva à suplementação de cobre ${ }^{45}$. Em nosso meio há relato de baixa ingestão de zinco em crianças e adolescentes de baixo nível econômico ${ }^{46}$, provavelmente pelo fato dos alimentos-fonte em zinco serem os de origem animal, que apresentam maior custo. Assim sendo, a anemia apresentada pelos indivíduos estudados não é conseqüente da interação entre ferro, cobre e zinco, mas provavelmente pela baixa ingestão de ferro e/ou baixa biodisponibilidade do ferro dietético. 


\section{ConCLUSÃo}

Os resultados obtidos permitem concluir que na população estudada, caracterizada pelo baixo nível econômico e institucionalização, ocorre o processo de transição nutricional e alta prevalência de anemia, que não resulta da interação ferro, cobre e zinco. Em ambas as situações podem ocorrer implicações futuras como menor desempenho profissional na idade adulta, associado com doenças crônicas não transmissíveis. Faz-se necessárias ações que visem a promoção e a prevenção da obesidade e de anemia na infância e adolescência.

\section{Conflito de interesse: não há.}

\section{SUMMARY}

\section{Nutritional status, iron, COPPER, and Zinc in SCHOOL CHILdREN OF SHANTYTOWNS OF Sao Paulo}

OBJECTIVE. To assess the anthropometry, body composition and iron, copper and zinc nutritional status, according to gender, of institutionalized children and adolescents living in two shantytowns in the city of Sao Paulo.

Methods. A cross sectional study using weight, height, arm circumference, skinfolds, electrical bioimpedance, $Z$ scores for the relationships: height to age, body mass index, middle-upper arm circumference, muscle area of the arm and fat area of the arm was carried out; the body fat and lean mass percentages were analyzed according to the formulas proposed by Siri and Slaughter. Hemoglobin, hematocrit, serum iron ferritin, copper and zinc were determined.

RESULTS. Body weight, middle-upper arm circumference, triceps and subescapular skinfolds, electric resistance, $Z$ scores of the arm area, muscle area of the arm, and body fat percentage of girls were higher in relation to boys. Low stature was found in $8 \%$ of the girls and in $5.6 \%$ of the boys, without differences according to gender. There was a lower prevalence of malnutrition (2\% of the girls and $5.6 \%$ of the boys), than of overweight and obesity (30\% and $11.2 \%$, respectively). Anemia was observed in $24.4 \%$ and iron deficiency in $10.5 \%$ of the schoolchildren with or without anemia. Values were below the lower limit of the reference standard for serum copper and zinc, respectively, for 3 and 7 individuals.

CONCLUSION. In the studied population, institutionalized and of low social economic level, a process of nutritional transition and high prevalence of anemia takes place which does not result from an interaction of iron, copper and zinc. [Rev Assoc Med Bras 2007; 53(4): 323-8]

KEY WORDS: Nutritional status. Body composition. Iron. Copper. Zinc.

\section{REFERÊNCIAS}

I. Onis M, Frongillo EA, Blössner M. Is malnutrition declining? An analysis of changes in levels of child malnutrition since 1980. Bull World Health Organ. 2000; 78(10): 1222-33.

2. Hoffman DJ, Sawaya AL, Andrew Coward W, Wright A, Martins AP,
Nascimento C, et al. Energy expenditure of stunted and nonstunted boys and girls living in the shantytowns of São Paulo. Brazil. Am J Clin Nutr. 2000;72:1025-31.

3. Wang Y, Monteiro CA, Popkin BM. Trends of obesity and underweight in older children and adolescents in the United States, Brazil, China, and Russia. Am J Clin Nutr. 2002;75:971-77.

4. Guimarães LV, Barros MBA. As diferenças do estado nutricional em préescolares de rede pública e a transição nutricional. J Pediatr (Rio de J). 2001;77(5):381-86

5. Sigulem DM, Devincenzi UM, Lessa AC. Diagnóstico do estado nutricional da criança e do adolescente. J Pediatr (Rio de J). 2000;76 (Supl):S275-S84.

6. Tsuyuoka R, Baley JW, D'Ávila AMNG, Gurgel RQ, Cuevas LE. Anemia and intestinal parasitic infections in primary school students in Aracaju, Sergipe, Brazil. Cad Saúde Publica. 1999;|5(2):4|3-2|

7. Bottoni A, Ciolette A, Schmitz BAS, Campanaro CM, Accioly E, Cuvello LCF. Anemia ferropriva. Rev Paul Pediatr. 1997; I5(3): 127-34.

8. Chacón R, Cordero M RE, Infante RB, Fajardo M, Hevia P. Composición corporal y concentración de cinc y cobre plasmático en ninõs sanos y desnutridos. Arch Venez Pueric Pediatr. 1998;6I(4):165-69.

9. Oski FA. Is bovine milk a health hazard? Pediatrics 1985;75(I):82-6.

10. Pedrazzani ES, Mello DA, Pripas S, Fucci M, Barbosa CAA, Santoro MCM. Helmintoses Intestinais. II: Prevalência e correlação com renda, tamanho da família, anemia e estado nutricional. Rev Saúde Publica. 1988;22(5):384-89.

II. Jellife DB. Evaluación del estado de nutrición de la comunidad. Genebra: Organización Mundial de la Salud; 1968. [Série de Monografias, 53].

12. Dean AG. Epi Info and Epi Map: planed for Epi Info 2000. J Public Health Manag Pract. 1999;5(4):54-7.

13. Himes JH, Dietz WH. Guidelines for overweight in adolescent preventive services: recommendations from an expert committee. Am J Clin Nutr. 1994;59(3):307-16.

14. Frisancho AR. Triceps skinfold and upper arm muscle size norms for assessment of nutritional status. Am J Clin Nutr. 1974;27(9): 1052-8.

15. Frisancho AR. Anthropometric standards for the assessment of growth and nutritional status. Ann Arbor: The University of Michigan Press; 1990. p.48-53.

16. Fjeld CR, Freundt-Thurne J, Schoeller DA. Total body water measured by ${ }^{18} 0$ dilution and bioelectrical impedance in well and malnourished children. Ped Res. 1990;27(10):98-102.

17. Siri WE. Tecniques for measuring body composition. Washington (DC): National Academy Press; 1961.

18. Slaughter MH, Lohman TG, Boileau RA, Horswill CA, Stillman RJ, Van Loan MD, et al. Skinfold equations for estimation of body fatness in children and youth. Hum Biol. 1988;60(5):709-23.

19. Brook CGD. Determination of body composition of children from skinfold measurements. Arch Dis Chid. 1971;46(2): 182-4.

20. Fomon SJ, Haschke F, Ziegler E, Nelson SE. Body composition of reference children from birth to age 10 years. Am J Clin Nutr. 1982;35(10):1169-75.

21. Hascke F. Body composition during adolescence. In: Klish WJ, Kretchmer $\mathrm{N}$. Body composition measurements in infants and children. Report of 98 $8^{\text {th }}$ Ross Conference on Pediatric Research. Columbus: Ross Laboratories; 1989. p.76-82.

22. Wordl Health Organization. Iron deficiency anaemia. Assessment, prevention, and control. A guide for programme managers. Ginebra: WHO; 200I.

23. Mabry CC, Tetz NW. Quadro com valores normais. In: Vaughan VC, McKay RJ, Behrman RE. Tratado de pediatria $2^{a}$ ed. Rio de Janeiro: Interamericana; 1983. p.|961-79.

24. Gibson RS. Assessment of trace-element status. In: Gibson RS. Principles of nutritional assessment. New York: Oxford University Press; 1990. p.522-5I

25. IBGE. Instituto Brasileiro de Geografia e Estatística. Síntese de indicadores sociais 2002/ IBGE, Departamento de População e Indicadores Sociais. Rio de Janeiro: IBGE; 2003.

26. Gross R, Lima FD, Freitas CJ, Gross U. The relationships between selected anthropometric and socio-ecomomic data in schoolchildren 
from different social strata in Rio de Janeiro, Brazil. Rev Saúde Publica 1990;24(I): I I-9.

27. Giugliano R, Melo ALP. Diagnóstico de sobrepeso e obesidade em escolares: utilizando índice de massa corporal segundo padrão internacional. J Pediatr (Rio de J). 2004;80(2): I29-34.

28. Abrantes MM, Lamounier JA, Colosimo EA. Prevalência de sobrepeso e obesidade em crianças e adolescentes das regiões Sudeste e Nordeste. J Pediatr (Rio de J). 2002;78(4):335-40.

29. Ruxton CHS, Reilly JJ, Kirk TR. Body composition of healthy 7 and 8 year-old children and a comparison with the "reference child". Int J Obes 1999;23(I |):|276-8|.

30. Boye KR, Dimitriou T, Manz F, Schoenau E, Neu C, Wudy S, et al. Anthropometric assessment of muscularity during growth: estimating fat-free mass with 2 skinfold-thickness measurements is superior to measuring midupper arm muscle area in healthy prepubertal children. Am J Clin Nutr. 2002;76(3):628-32.

31. Berkey CS, Rockett HR, Field AE, Gilman MW, Frazier AL, Camargo CAJRT. Activity, dietary intake, and weight changes in a longitudinal study of preadolescent and adolescent boys and girls. Pediatrics. 2000; I 05(4):E56.

32. Bundred P, Kitchiner D, Buchan I. Prevalences of overweight and obese children between 1989 and 1998; population based series of crosssectional studies. Br Med J. 2001;322:I-4.

33. Parsons TJ, Power C, Logan S, Summerbell CD. Childhood predictors of adult obesity: a systematic review. Int J Obes Relat Metab Disord. 1999;23(Suppl 8): I- I07.

34. Dutra CL, Araújo CL, Bertoldi AD. Prevalence of overweight in adolescents: a population-based study in a southern Brazilian city. Cad Saúde Publica 2006;22(1):151-62.

35. Monteiro CA, Szarfarc SC, Mondini L. Tendência secular da anemia na infância na cidade de São Paulo (1984-1996). Rev Saúde Publica 2000;34( Supl):S62-S72.

36. Rocha YR, Yuyama LKO, Nascimento OP. Perfil nutricional de préescolares e escolares residentes em Palmeiras do Javari, AM. Acta Amaz. 1993;23(1):9-14.

37. Norton RC, Figueiredo RCP, Diamante R, Goulart EMA, Mota JAC, Viana MB, et al. Prevalence of anemia among school-children from Rio Acima (State of Minas Gerais, Brazil): use of the standardized prevalence method and evaluation of iron deficiency. Braz J Med Biol Res. 1996;29(12): 1617-24
38. Alarcón OM, Fuller JR, Silva TM, Angarita C, Terán E, Navas M, et al. Serum level of Zn, Cu and Fe in healthy schoolchildren residing in Mérida, Venezuela. Arch Latinoam Nutr. 1997;47(2):1।8-22.

39. Nead KG, Halterman JS, Kaczorowski JM, Auinger P, Weitzman M. Overweight children and adolescents: a risk group for iron deficiency. Pediatrics. 2004; I|4(I): 104-8.

40. Estevéz J, Bonilha LC, Bonilha E, Villalobos R. Concentraciones sericas de cobre y cinc en una población suburbana del estado Zulia (Venezuela). Invest Clin. 1988;29(3):97-109.

4I. Laitinen R, Räsänen L, Vuori E. Serum zinc and copper in relation to diet in 3- to 18-year old finnish girls and boys. Eur J Clin Nutr. 1988;42(11):911-8.

42. Fávaro RMD, Vannucchi H. Níveis plasmáticos de zinco e antropometria de crianças da periferia de centro urbano no Brasil. Rev Saúde Publica 1990;24(1):5- 10.

43. Sandstead $\mathrm{HH}$. Copper biovailability and requirements. Am J Clin Nutr. 1982;35(8):809-। 4.

44. Harris ED. Iron-copper interactions: some new revelations. Nutr Rev. 1994;52(9):3। I- I 5.

45. Hill $\mathrm{CH}$, Matrone G. Chemical parameters in the study of in vivo and in vitro interactions of transition elements. Fed Proc. 1970;29(4): | 474-8|.

46. Urbano MRD, Vitalle MSS, Juliano Y, Amancio OMS. Ferro, cobre e zinco em adolescentes no estirão pubertário. J Pediatr (Rio de J). 2002;78(4):327-34. 